\title{
The association of ischaemic stroke in patients with heart failure without atrial flutter/fibrillation
}

\author{
Yen-Lien Chou, ${ }^{1}$ Jun-Ting Liou, ${ }^{1}$ Cheng-Chung Cheng, ${ }^{1}$ Min-Chien Tsai, ${ }^{2}$ \\ Wei-Shiang Lin, ${ }^{1}$ Shu-Meng Cheng, ${ }^{1}$ Cheng-Li Lin, ${ }^{3}$ Chin-Sheng Lin, ${ }^{1}$ \\ Chia-Hung Kao (1) 3,4,5
}

'Division of Cardiology, Department of Medicine, Tri-Service General Hospital, National Defense Medical Center, Taipei, Taiwan ${ }^{2}$ Department of Physiology and Biophysics, Graduate Institute of Physiology, National Defense Medical Center, Taipei, Taiwan ${ }^{3}$ Graduate Institute of Biomedical Sciences and School of Medicine College of Medicine, China Medical University, Taichung, Taiwan ${ }^{4}$ Department of Nuclear Medicine and PET Center Center of Augmented Intelligence in Healthcare China Medical University Hospital, Taichung, Taiwan ${ }^{5}$ Department of Bioinformatics and Medical Engineering, Asia University, Taichung, Taiwan

\section{Correspondence to}

Dr Chia-Hung Kao, China

Medical University, Taichung

404, Taiwan;

d10040@mail.cmuh.org.tw

Dr Chin-Sheng Lin;

littlelincs@gmail.com

C-SL and C-HK contributed equally.

Received 9 July 2019

Revised 2 September 2019

Accepted 10 September 2019

Published Online First

3 October 2019

\section{Linked}

- http://dx.doi.org/10.1136/ heartjnl-2019-315957

\section{Check for updates}

(c) Author(s) (or their employer(s)) 2020. No commercial re-use. See rights and permissions. Published by BMJ.

To cite: Chou Y-L, Liou J-T, Cheng C-C, et al. Heart 2020;106:616-623.

\section{ABSTRACT \\ Purpose This study evaluated the association between} ischaemic stroke (IS) and heart failure (HF) in the absence of atrial fibrillation (AF) or atrial flutter (AFL) using a population-based nation-wide cohort database. Method Newly diagnosed patients with HF without previous stroke and acute myocardial infarction (AMI) were enrolled. Based on the propensity scores matching age, sex and all comorbidities, our studies comprised 12179 patients with HF and 12179 patients without HF. Cox proportion hazard regression models and competing-risk regression models were used to evaluate the risk of IS among patients with HF without AF or AFL.

Results In the multivariable analysis, older age (adjusted HR $(95 \% \mathrm{Cl})=1.05$ (1.04 to 1.05)), male sex (adjusted HR $(95 \% \mathrm{Cl})=1.36(1.24$ to 1.50$))$, diabetes (adjusted HR $(95 \% \mathrm{Cl})=2.22(1.97$ to 2.49$))$ and hypertension (adjusted HR $(95 \% \mathrm{Cl})=1.60(1.41$ to 1.82)) were markedly associated with IS in patients with HF. The HF group had a markedly higher risk of IS than did the non-HF group (subdistribution HR $(S H R)=1.51$, 95\% Cl: 1.37 to 1.66) and AMI (SHR=3.40,95\% Cl: 2.71 to 4.28). Additionally, according to the Kaplan-Meier analysis, patients with HF were at a significantly higher risk of cumulative incidence of IS and AMI than did patients with non-HF ( $p$ value of log-rank test $<0.001$ ). Conclusion This study indicated that $\mathrm{HF}$ is a strong independent risk factor for IS, even in the absence of $\mathrm{AF}$ or AFL. Clinical physicians should investigate IS through routine screening and careful monitoring of patients with HF.

\section{INTRODUCTION}

Heart failure (HF) is a widespread medical condition and a major cause of hospitalisation, death and comorbidity worldwide. ${ }^{1} \mathrm{HF}$ is a complex clinical syndrome characterised by dysfunction of diastole or systole of the ventricle through either structural or functional abnormalities. Common predisposing factors of HF include hypertension, valvular heart disease, diabetes, atrial fibrillation (AF), coronary artery disease (CAD) and alcohol consumption..$^{2-4}$ Currently, the latest evidence-based medical treatment for HF has markedly improved the survival rate, major adverse cardiovascular events (MACE) and re-hospitalisation. However, the risk of stroke among patients with HF has considerably increased than in the past. ${ }^{1}$ Moreover, the risk of silent stroke, which is defined as ischaemic brain lesions without obvious clinical symptoms, is two to three times higher in patients with HF than in those without $\mathrm{HF}^{5-7}$ Such evidence highlights the increasing disease burden of stroke in patients with HF even under promising therapeutic medications for HF.

Patients with ischaemic stroke (IS) have a long hospitalisation duration, poor quality of life, disability, long-term complications and a high probability of mortality. Additionally, IS has been the leading cause of hospitalisation, mortality, disability and comorbidity worldwide. Risk factors for IS are similar to those of HF, including diabetes mellitus, hypertension, obesity, CAD and AF. ${ }^{15} 8$ Patients with simultaneous HF and IS have more severe neurological deficits than those with stroke only. ${ }^{156} \mathrm{HF}$ is a definite risk factor in $\mathrm{AF}$ to predict the probability of IS within the $\mathrm{CHA}_{2} \mathrm{DS}_{2}$-VACs score. $^{5}$ Nearly $10 \%-25 \%$ of patients with IS have HF. AF occurs in $57 \%$ of patients with HF, which contributes the most to the incidence of stroke. ${ }^{7910}$ The coexistence of HF and AF brings the synergistic risk of IS development, which is not as prevalent in the presence of either HF or AF. These results summarise the vital roles of AF in patients with IS and $\mathrm{HF}$

Few studies have evaluated the association of IS with HF in patients without AF or atrial flutter (AFL). Most of these studies have a small sample size and did not evaluate confounding factors including incident AF/AFL and anticoagulants. ${ }^{7} 1011$ Therefore, we conducted a national population-based cohort study to evaluate the risk of IS in patients with HF without AF or AFL and identified potential risk factors linking these two diseases, which is beneficial for further stroke prevention in patients with HF but without AF or AFL.

\section{METHODS}

\section{Data source}

The extent of insured Taiwanese population under the Taiwan National Health Insurance programme (Taiwan NHI) was over 99\%. The Taiwan National Health Insurance Database (NHIRD) was established and updated once a year from 1996. We retrieve data from LHID2000. The LHID2000 contains all medical claims data for a random sampling of 1000000 beneficiaries from the whole insurants (23.74 million people). According to the NHRID, no statistically significant differences exist regarding the distribution of sex, age or healthcare costs between cohorts in LHID2000 and all 
insurants. The database contains abundant health and medical treatment information of insured individuals including their diagnoses history in a coded of the International Classification of Disease, Ninth Revision, Clinical Modification (ICD-9-CM).

\section{Patient and public involvement}

In the HF cohort, patients newly diagnosed with HF (ICD-9-CM code 428) between January 2000 and December 2012 were enrolled. The date of the first diagnosis was defined as the index date. We excluded patients with AF (ICD-9-CM code 427.31) and AFL (ICD-9-CM code 427.32) who were diagnosed before the index date or during the follow-up period. Additionally, we excluded patients with a previous history of stroke (ICD-9-CM codes 430-438) and acute myocardial infarction (AMI; ICD-9-CM code 410) at baseline.

We used propensity score for selecting the non-HF cohort by matching age, sex, monthly income, urbanisation level and certain comorbidities. ${ }^{12}$ Patients with HF were matched $(1: 1$ ratio) with those without HF by their propensity score through nearest neighbour matching, initially to the eighth digit and then as required to the first digit. Therefore, matches were first made within a calliper width of 0.0000001 , and then the calliper width was increased for unmatched cases to 0.1 . We reconsidered the matching criteria and performed a rematch (greedy algorithm). For each patient with HF, the corresponding comparisons were selected based on the nearest propensity score. The C-statistic of the logistic regression model was 0.75 . The presence of the following pre-existing comorbidities for each patient were identified at baseline: diabetes (ICD-9-CM code 250), hypertension (ICD-9-CM codes 401-405), hyperlipidaemia (ICD-9-CM code 272), chronic kidney disease (CKD) (ICD-9-CM codes 250.4, 274.1, 283.11, 403.1, 404.2, 404.3, 404.1, 442.1, 447.3,
$580-583,585,587,792.5,642.1$ and 646.2), chronic obstructive pulmonary disease (ICD-9-CM codes 490-496), peripheral vascular disease (ICD-9-CM codes 440.20-440.24, 440.9, 443.81, 443.9 and 444.22), alcohol-related illness (ICD-9-CM codes 291, 303, 305, 357.5, 425.5, 535.3, 571 and E860.0), mild liver diseases (ICD-9-CM codes 571.2, 571.3, 571.5 and 573.8), obesity (ICD-9-CM code 278.0), autoimmune disease (ICD-9-CM codes 135, 279.49, 283, 443, 571.42, 696, 710, 714 and 715), depression (ICD-9-CM codes 296.2, 296.3, 300.4 and 311 ), anxiety (ICD-9-CM code 300), all cancer types (ICD-9-CM codes 140-208), trauma (ICD-9-CM codes 365.65, 366.2, 376.47, 376.52, 380.51, 381.1, 518.5, 598.1, 664.8, $664.9,665,665.8,665.9,716.1,721.7,728.12,860,878,885$, $886,887,895,896,897,958,958.4,958.5,958.7$ and 958.8) and CAD (ICD-9-CM codes 411-414). The same exclusion criteria were used for non-HF cohort (figure 1).

The endpoint for follow-up was the date of withdrawal from the programme in patients who developed one of the outcome diseases, namely IS (ICD-9-CM codes 433-435), haemorrhagic stroke (HS) (ICD-9-CM codes 430-432) and AMI. The follow-up endpoint in patients without event was the last follow-up date (31 December 2013).

\section{Statistical analysis}

Distributions of age, sex, monthly income, urbanisation level and comorbidities in HF and non-HF cohorts are shown in numbers and percentage. The difference between the two cohorts was tested by calculating standardised mean differences, in which a value less than 0.1 indicated a negligible difference between the two cohorts. HRs and 95\% CIs were estimated using Cox proportional hazard models for evaluating the association of $\mathrm{HF}$ with stroke, IS, HS and AMI. To address the concern of constant

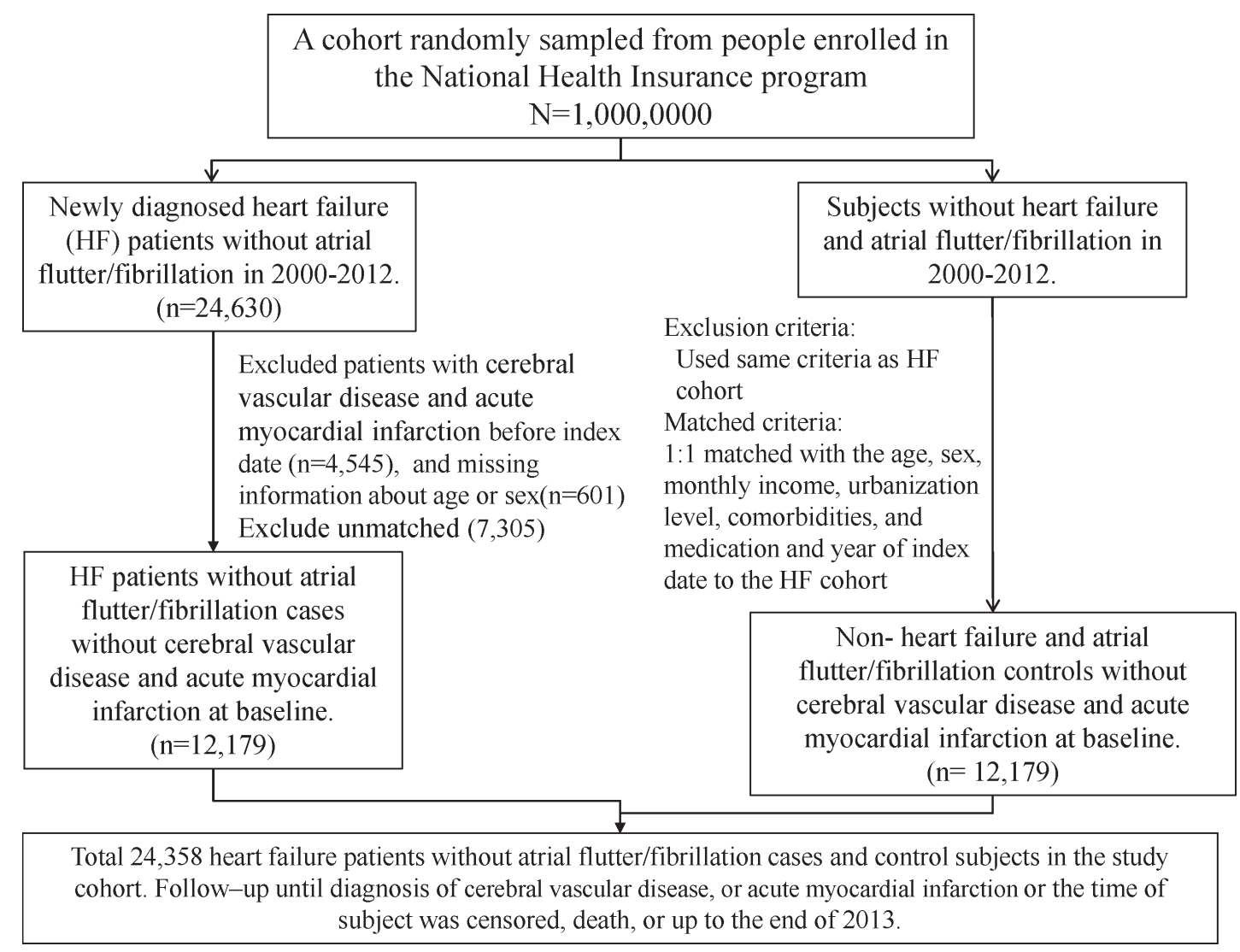

Figure 1 Flow chart of the study design. 
Table 1 Baseline characteristics of patients, by the presence of $\mathrm{HF}$, and after matching for propensity score

\begin{tabular}{|c|c|c|c|}
\hline & \multirow{2}{*}{$\begin{array}{l}\text { Without HF } \\
n=12179\end{array}$} & \multirow{2}{*}{$\begin{array}{l}\text { With HF } \\
n=12179\end{array}$} & \multirow{2}{*}{$\begin{array}{l}\text { Standardised } \\
\text { mean } \\
\text { difference }^{*}\end{array}$} \\
\hline & & & \\
\hline \multicolumn{4}{|l|}{ Age, years } \\
\hline$\leq 49$ & $1402(11.5)$ & $1771(14.5)$ & 0.09 \\
\hline $50-64$ & $2895(23.8)$ & $3187(26.2)$ & 0.06 \\
\hline $65+$ & $7882(64.7)$ & $7221(59.3)$ & 0.11 \\
\hline Mean \pm SD & $67.0(13.9)$ & $66.6(14.7)$ & 0.03 \\
\hline \multicolumn{4}{|l|}{ Sex } \\
\hline Female & $6672(54.8)$ & $6651(54.6)$ & 0.003 \\
\hline Male & $5507(45.2)$ & $5528(45.4)$ & 0.003 \\
\hline \multicolumn{4}{|l|}{ Monthly income (NTD) } \\
\hline$<15000$ & 4374 (35.9) & $4214(34.6)$ & 0.03 \\
\hline 15000-19999 & $3246(26.7)$ & $3668(30.1)$ & 0.08 \\
\hline$\geq 20000$ & 4559 (37.4) & $4297(35.3)$ & 0.045 \\
\hline \multicolumn{4}{|l|}{ Urbanisation level } \\
\hline 1 (highest) & $3538(29.1)$ & $3263(26.8)$ & 0.05 \\
\hline 2 & $3416(28.1)$ & $3290(27.0)$ & 0.02 \\
\hline 3 & $1898(15.6)$ & $1864(15.3)$ & 0.01 \\
\hline 4 (lowest) & $3327(27.3)$ & $3762(30.9)$ & 0.08 \\
\hline \multicolumn{4}{|l|}{ Comorbidity } \\
\hline Diabetes & $1451(11.9)$ & $1457(12.0)$ & 0.002 \\
\hline Hypertension & $8398(69.0)$ & $8149(66.9)$ & 0.04 \\
\hline Hyperlipidaemia & 4599 (37.8) & $4526(37.2)$ & 0.01 \\
\hline Chronic kidney disease & $2164(17.8)$ & $2221(18.2)$ & 0.01 \\
\hline $\begin{array}{l}\text { Chronic obstructive } \\
\text { pulmonary disease }\end{array}$ & $4900(40.2)$ & $4842(39.8)$ & 0.01 \\
\hline Peripheral vascular disease & $730(5.99)$ & $727(5.97)$ & 0.001 \\
\hline Alcohol-related illness & $419(3.44)$ & $416(3.42)$ & 0.001 \\
\hline Mild liver diseases & $426(3.50)$ & $428(3.51)$ & 0.001 \\
\hline Obesity & $187(1.54)$ & $192(1.58)$ & 0.003 \\
\hline Autoimmune disease & $5696(46.8)$ & $5637(46.3)$ & 0.01 \\
\hline Anxiety & $3406(28.0)$ & $3354(27.5)$ & 0.01 \\
\hline Depression & $884(7.26)$ & $830(6.82)$ & 0.02 \\
\hline Cancer & $613(5.03)$ & $572(4.70)$ & 0.02 \\
\hline Trauma & $460(3.78)$ & $483(3.97)$ & 0.01 \\
\hline Coronary artery disease & $1290(10.6)$ & $1475(12.1)$ & 0.048 \\
\hline \multicolumn{4}{|l|}{ Medication } \\
\hline Warfarin & $105(0.86)$ & $125(1.03)$ & 0.02 \\
\hline
\end{tabular}

proportionality, we examined the proportional hazard model assumption using a test of scaled Schoenfeld residuals. There was no significant relationship between Schoenfeld residuals for $\mathrm{HF}$ and follow-up time ( $\mathrm{p}$ value $=0.67$ ) in the model of evaluating IS. Interestingly, there was significant relationship between Schoenfeld residuals for HF and follow-up time in the models of evaluating stroke, HS and AMI ( $p$ value $=0.003,<0.0001$, 0.0001 , respectively). In the subsequent analyses, we stratified the follow-up duration to deal with the violation of proportional hazard assumption. A multivariate Cox proportional hazard model was used to estimate adjusted HRs after adjustment for age, sex, monthly income, urbanisation level and all comorbidities.

We used the Fine and Grey model which extends the standard Cox proportional hazard regression model to estimate the subdistribution HR of the stroke, IS, HS and AMI, after
Table 2 Incidence and HR of stroke, IS, HS and AMI in patients with atrial flutter compared with those without heart failure

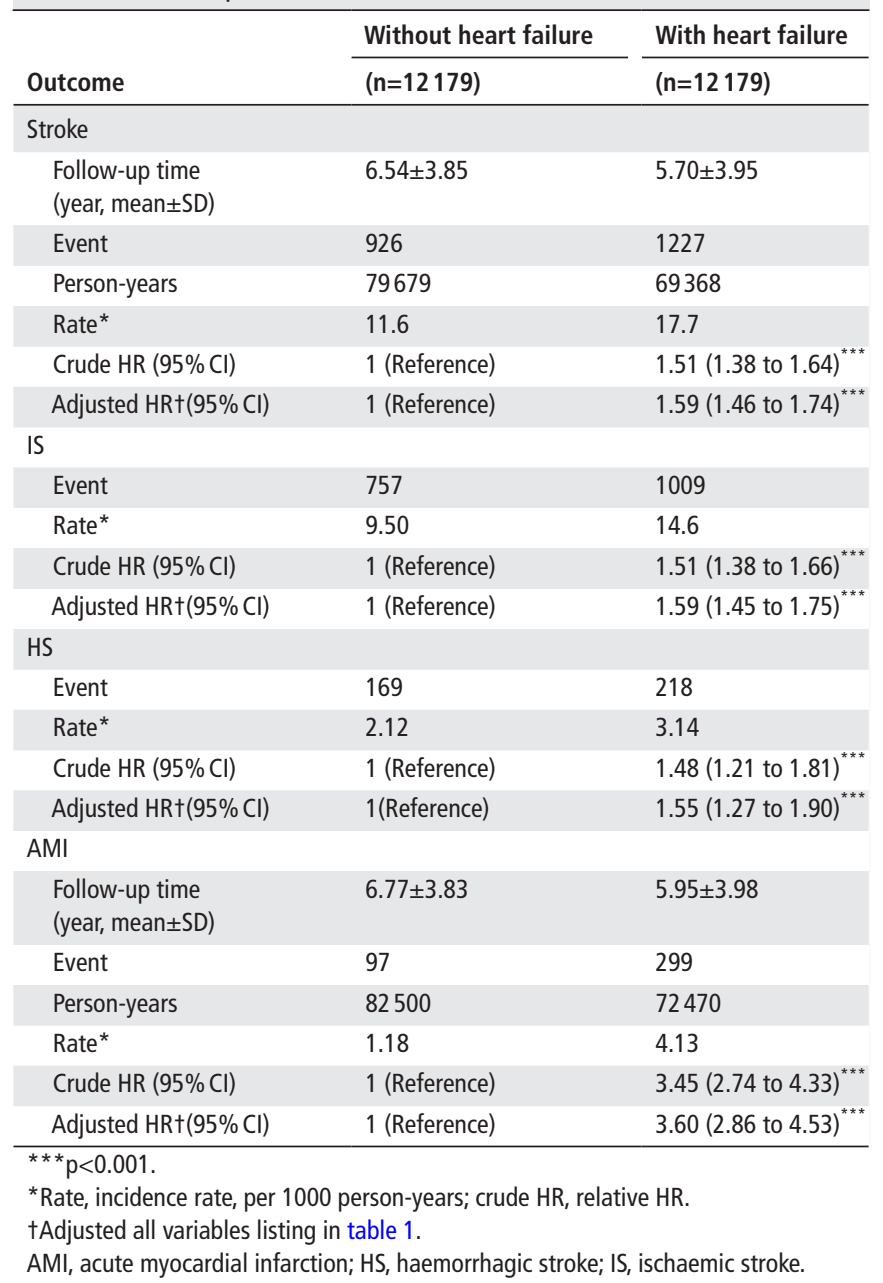

evaluating the competing risk of deaths. The subdistribution HR (SHR) and 95\% CIs were calculated using the competing-risk regression model. ${ }^{13}$ The cumulative incidence curves of stroke and AMI were plotted using the Kaplan-Meier method, and the difference between the HF and non-HF cohorts was tested using the log-rank test. All statistical analyses were performed using SAS statistical software, V.9.4 (SAS Institute, Cary, NC, USA). The Kaplan-Meier plot was plotted using R software. Statistical significance was determined using two-tailed tests $(\mathrm{p}<0.05)$.

\section{RESULTS}

The baseline characteristics of patients are shown in table 1. Each group consisted of 12179 patients (HF and non-HF cohorts). Except in the subgroup of patients older than 65 years, each subgroup was consistent in age, sex, monthly income, urbanisation level and all comorbidities of participants in the two cohorts (standardised mean difference $<0.1$ ).

Mean follow-up times were 6.54 and 5.70 years in the HF cohort and non-HF cohort, respectively. Compared with patients with non-HF, patients with HF had a significantly higher risk of stroke, IS, HS and AMI; adjusted HRs (95\% CI) were 1.59 (1.46-1.74), 1.59 (1.45-1.75), $1.55(1.27-1.90)$ and 3.60 (2.86-4.53), respectively (table 2 ).

The association of sex, age and comorbidities with IS, HS and AMI is shown in table 3. In multivariable models, old age (adjusted HR $(95 \% \mathrm{CI})=1.05(1.04$ to 1.05$))$, male sex (adjusted HR 


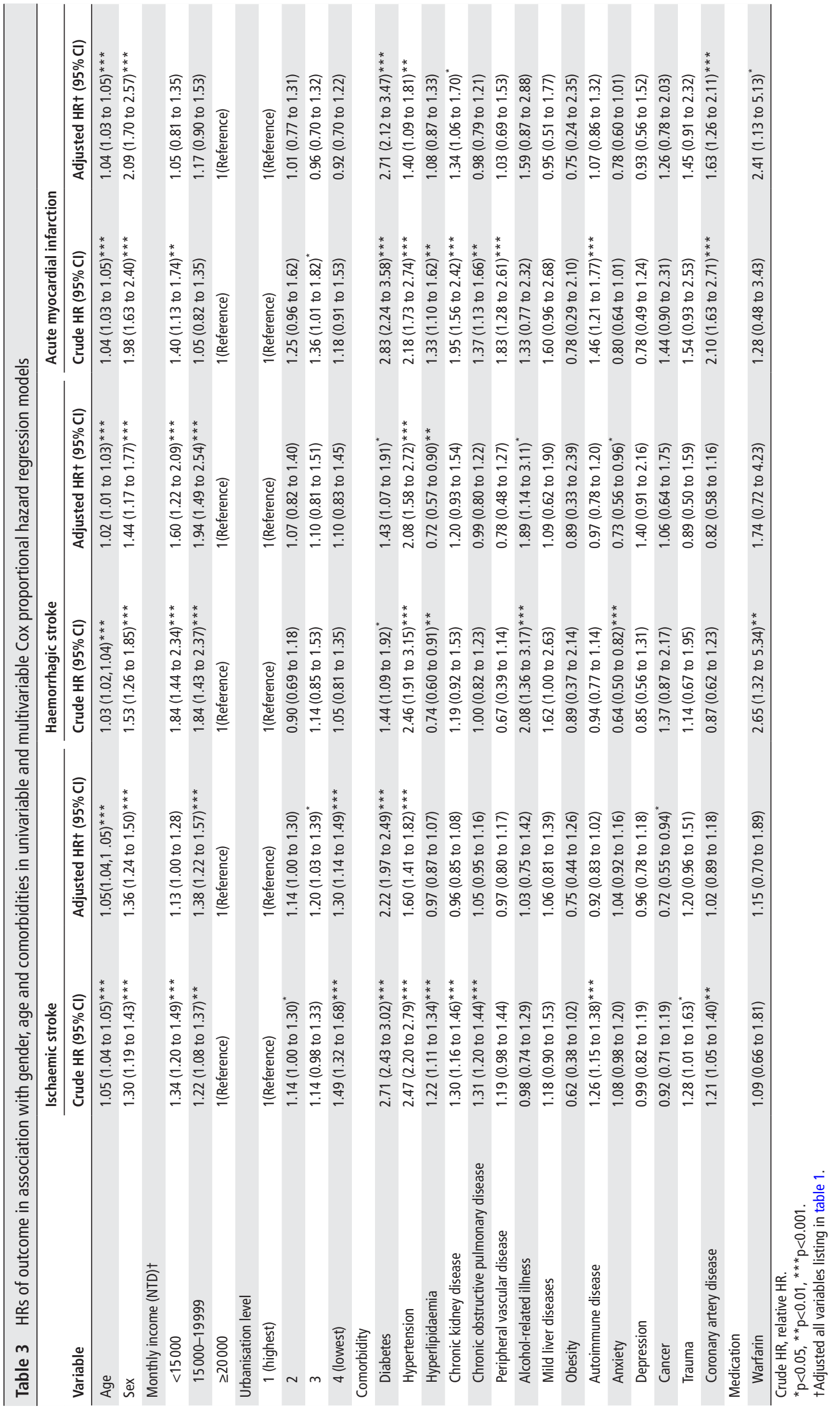


Table 4 Incidence and SHR of stroke, IS, HS, and AMI in propensity score-matched cohorts, using the competing-risks regression models

\begin{tabular}{|c|c|c|}
\hline \multirow[b]{2}{*}{ Outcome } & \multicolumn{2}{|c|}{ Competing-risks regression models } \\
\hline & $\begin{array}{l}\text { Without heart failure } \\
(n=12179)\end{array}$ & $\begin{array}{l}\text { With heart failure } \\
(n=12179)\end{array}$ \\
\hline \multicolumn{3}{|l|}{ Stroke } \\
\hline Crude SHR $(95 \% \mathrm{Cl})$ & 1 (Reference) & $1.46(1.40 \text { to } 1.59)^{* * *}$ \\
\hline Adjusted SHR $\ddagger(95 \% \mathrm{Cl})$ & 1 (Reference) & $1.50(1.38 \text { to } 1.63)^{* * *}$ \\
\hline \multicolumn{3}{|l|}{ IS } \\
\hline Crude SHR $(95 \% \mathrm{Cl})$ & 1 (Reference) & $1.46(1.39 \text { to } 1.61)^{* * *}$ \\
\hline Adjusted SHR $\ddagger(95 \% \mathrm{Cl})$ & 1 (Reference) & $1.51(1.37 \text { to } 1.66)^{\star * *}$ \\
\hline \multicolumn{3}{|l|}{ HS } \\
\hline Crude SHR $(95 \% \mathrm{Cl})$ & 1 (Reference) & $1.42(1.16 \text { to } 1.73)^{* * *}$ \\
\hline Adjusted SHR $¥(95 \% \mathrm{Cl})$ & 1 (Reference) & $1.45(1.19 \text { to } 1.77)^{* \star *}$ \\
\hline \multicolumn{3}{|l|}{ AMI } \\
\hline Crude SHR $(95 \% \mathrm{Cl})$ & 1 (Reference) & $3.34(2.66 \text { to } 4.19)^{* * *}$ \\
\hline Adjusted SHR $\ddagger(95 \% \mathrm{Cl})$ & 1 (Reference) & $3.40(2.71 \text { to } 4.28)^{* * *}$ \\
\hline \multicolumn{3}{|c|}{ 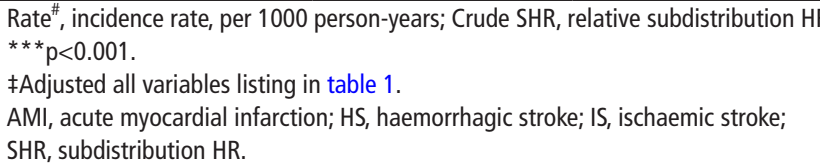 } \\
\hline
\end{tabular}

$(95 \% \mathrm{CI})=1.36$ [1.24 to 1.50$])$, lower monthly income (adjusted HR $(95 \% \mathrm{CI})=1.38(1.22$ to 1.57$))$, lower urbanisation level (adjusted HR $(95 \% \mathrm{CI})=1.30$ (1.14 to 1.49$)$ ), diabetes (adjusted $\operatorname{HR}(95 \% \mathrm{CI})=2.22(1.97$ to 2.49$))$ and hypertension (adjusted HR $(95 \% \mathrm{CI})=1.60(1.41$ to 1.82$))$ were significantly associated with IS. Patients with cancer (adjusted HR $(95 \% \mathrm{CI})=0.72$ (0.55 to 0.94$))$ had a significantly negative association with IS. Old age (adjusted HR $(95 \% \mathrm{CI})=1.02$ (1.01 to 1.03$)$ ), male sex (adjusted HR $(95 \% \mathrm{CI})=1.44(1.17$ to 1.77$))$, lower monthly income (adjusted HR $(95 \% \mathrm{CI})=1.94(1.49$ to 2.54$)$ ), diabetes (adjusted HR $(95 \% \mathrm{CI})=1.43(1.07$ to 1.91$))$, hypertension (adjusted HR $(95 \% \mathrm{CI})=2.08(1.58$ to 2.72$)$ ) and alcoholrelated illness (adjusted HR $(95 \% \mathrm{CI})=1.89$ (1.14 to 3.11$)$ ) were significantly associated with HS. Patients with hyperlipidaemia (adjusted HR $(95 \% \mathrm{CI})=0.72(0.57$ to 0.90$)$ ) or anxiety (adjusted HR $(95 \% \mathrm{CI})=0.73(0.56$ to 0.96$))$ had a significantly lower adjusted HR for HS. Old age (adjusted HR $(95 \% \mathrm{CI})=1.04$ (1.03 to 1.05$)$ ), male sex (adjusted HR $(95 \% \mathrm{CI})=2.09(1.70$ to 2.57)), comorbid diabetes (adjusted HR $(95 \% \mathrm{CI})=2.71(2.12$ to 3.47)), hypertension (adjusted HR $(95 \% \mathrm{CI})=1.40 \quad(1.09$ to 1.81$)$ ), CKD (adjusted $\mathrm{HR}(95 \% \mathrm{CI})=1.34(1.06$ to 1.70$)$ ), CAD (adjusted HR $(95 \% \mathrm{CI})=1.63(1.26$ to 2.11$))$ and warfarin (adjusted HR $(95 \% \mathrm{CI})=2.41$ (1.13 to 5.13$)$ ) were significantly associated with AMI.

Table 4 shows the adjusted SHR after considering death as a competing outcome. The HF group was compared with the non-HF group. After adjustment for all the variables listed in table 1 , there was a significantly higher risk of stroke $(\mathrm{SHR}=1.50$, $95 \% \mathrm{CI}: 1.38$ to 1.63 ), IS (SHR=1.51, 95\% CI: 1.37 to 1.66 ), HS (SHR $=1.45,95 \% \mathrm{CI}: 1.19$ to 1.77$)$ and AMI (SHR=3.40, 95\% CI: 2.71 to 4.28 ) in the HF group. The higher risk of stroke, HS and AMI occurred during the first 1 year of follow-up period in patients with HF compared with those without HF, while the trend reduced in the follow-up period of 2-4 years and $>5$ years (table 5). The cumulative incidences of stroke and AMI are illustrated in figure 2. Patients with HF had a significantly higher cumulative incidence of stroke and AMI than did those without HF during the follow-up periods ( $p$ value of log-rank test $<0.001)$.

\section{DISCUSSION}

In this study, the relationship among HF without AF or AFL, stroke, IS, HS and AMI were comprehensively investigated. Our results consistently exhibited HF as a significant independent risk factor for stroke, IS, HS and AMI after adjustment for comorbidities and medication with warfarin. Risk factors for IS among patients with HF without AF or AFL were old age, male sex, low urbanisation level and diabetes. Among these, diabetes was the strongest risk factor for IS and AMI in patients with HF without AF or AFL. These results indicate that HF is an independent risk factor for IS and AMI even in the absence of AF or AFL. Our study findings remind both physicians and patients to carefully screen for IS and AMI in patients with HF without AF or AFL, especially those with diabetes.

Table 5 Incidence ratio and HR of stroke, HS and AMI in patients with/without heart failure stratified by follow-up period

\begin{tabular}{|c|c|c|c|c|c|c|c|c|}
\hline \multirow[b]{3}{*}{ Follow time, years } & \multicolumn{6}{|c|}{ Heart failure } & \multirow[b]{3}{*}{ Crude HR $(95 \% \mathrm{CI})$} & \multirow[b]{3}{*}{ Adjusted HR† $(95 \% \mathrm{Cl})$} \\
\hline & \multicolumn{3}{|l|}{ No } & \multicolumn{3}{|l|}{ Yes } & & \\
\hline & Event & PY & Rate* & Event & PY & Rate* & & \\
\hline \multicolumn{9}{|l|}{ Stroke } \\
\hline$\leq 1$ & 161 & 11929 & 13.5 & 293 & 11441 & 25.6 & $1.89(1.56 \text { to } 2.29)^{* * *}$ & $1.92(1.58 \text { to } 2.33)^{* * *}$ \\
\hline $2-4$ & 446 & 37516 & 11.9 & 576 & 33293 & 17.3 & $1.45(1.29 \text { to } 1.65)^{* * *}$ & $1.54(1.36 \text { to } 1.74)^{* * *}$ \\
\hline$>5$ & 319 & 30234 & 10.6 & 358 & 24634 & 14.5 & $1.38(1.19 \text { to } 1.60)^{* * *}$ & $1.49(1.28 \text { to } 1.74)^{* * *}$ \\
\hline \multicolumn{9}{|l|}{ HS } \\
\hline$\leq 1$ & 133 & 11929 & 11.2 & 255 & 11441 & 22.3 & $1.99(1.62 \text { to } 2.46)^{* * *}$ & $2.03(1.64 \text { to } 2.50)^{* * *}$ \\
\hline $2-4$ & 369 & 37516 & 9.84 & 469 & 33293 & 14.1 & $1.36(1.19 \text { to } 1.56)^{* * *}$ & $1.41(1.23 \text { to } 1.62)^{* * *}$ \\
\hline$>5$ & 255 & 30234 & 8.43 & 285 & 24634 & 11.6 & $1.20(1.01 \text { to } 1.42)^{*}$ & $1.21(1.03 \text { to } 1.44)^{*}$ \\
\hline \multicolumn{9}{|l|}{ AMI } \\
\hline$\leq 1$ & 12 & 11993 & 1.00 & 94 & 11521 & 8.16 & $8.05(4.42 \text { to } 14.7)^{* * *}$ & $8.19(4.49 \text { to } 14.9)^{* * *}$ \\
\hline $2-4$ & 39 & 38477 & 1.01 & 127 & 34381 & 3.69 & $3.63(2.53 \text { to } 5.19)^{* * *}$ & $3.81(2.66 \text { to } 5.46)^{* * *}$ \\
\hline$>5$ & 46 & 32030 & 1.44 & 78 & 26568 & 2.94 & $2.04(1.42 \text { to } 2.94)^{* * *}$ & $2.17(1.50 \text { to } 3.13)^{* * *}$ \\
\hline
\end{tabular}

${ }^{*}$ Rate, incidence rate, per 1000 person-years; Crude HR, relative HR.

tAdjusted all variables listing in table 1.

$\ddagger$

$\S$

AMI, acute myocardial infarction; HS, haemorrhagic stroke; HS, haemorrhagic stroke. 
(A)

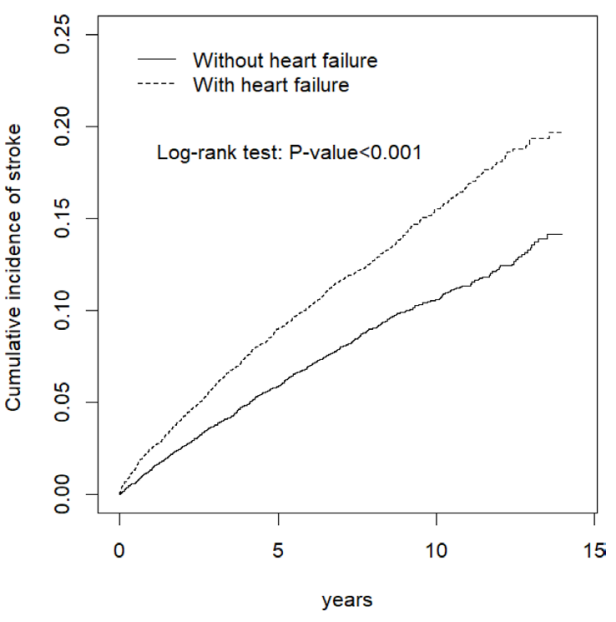

Heart failure, No.

At risk

Stroke events

Non-heart failure, No.

At risk

Stroke events

(B)

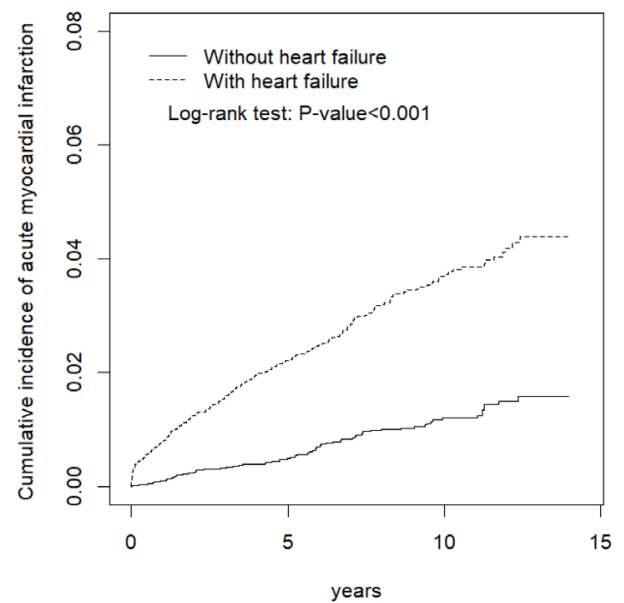

Heart failure, No.

At risk

Acute myocardial

infarction events

Non-heart failure, No.

At risk

Acute myocardial

infarction events
12179

0

12179

0
6065
869

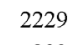

293

2816

263

56

\section{5}

$\begin{array}{rrrr}12179 & 6423 & 2438 & 0 \\ 0 & 221 & 67 & 11 \\ 12179 & 7522 & 3009 & 0 \\ 0 & 51 & 39 & 7\end{array}$

Figure 2 Cumulative incidence of ischaemic stroke $(A)$ and acute myocardial infarction (B) in individuals with $\mathrm{HF}$ or without $\mathrm{HF}$ by propensity score matching. $\mathrm{HF}$, heart failure.

In this study, we used the nation-wide population-based cohort from the NHIRD, which includes all Taiwanese residents. The rate of lost to follow-up was low in the longitudinal design, including both study and control cohorts. Additionally, due to the universal reimbursement policy in Taiwan, all insurance claims should be evaluated according to the standard diagnosed criteria by medical reimbursement specialists. The doctors or hospitals will be punished with huge penalties when they make wrong diagnoses or coding. Such polices contribute to the highly reliable diagnoses of IS, HF, AF/AFL based on ICD-9 codes in this study.

Several pathophysiologic mechanisms underlying stroke and HF have been proposed. In previous studies, AF and left ventricular (LV) dysfunction were the most commonly pathophysiologic mechanisms underlying thrombus formation. ${ }^{10} 14 \mathrm{HF}$ with or

without LV dysfunction containing Virchow's triad (abnormalities with circulatory stasis, hypercoagulable state and endothelial abnormality) contributes to the development of stroke. ${ }^{1516}$ First, the hypercoagulable state among patients with HF with activation of the renin-angiotensin-aldosterone system and increasing the sympathetic nerve system results in exaggeration of platelet aggregation and hypofibrinolysis. ${ }^{15} 17$ Second, endothelial abnormality among patients with HF due to common risk factors of hypertension and diabetes is significantly associated with high von Willebrand factor levels and impairment of endotheliumderived nitric oxide (NO) leading to hypercoagulable condition. ${ }^{15} 16$ Third, circulatory stasis resulting from enlargement of the left atrium or ventricle and systolic dysfunction contribute to thrombus formation. ${ }^{101819}$ Furthermore, hypoperfusion of cerebral blood flow in patients with HF with decreasing cardiac output and cerebral autoregulation dysfunction is a documented mechanism for stroke. Finally, elevation of arterial stiffness and reduction of vasodilatory reserve with reducing cerebral blood flow are proposed as pathological mechanisms for stroke. ${ }^{20} 21$ All results including abnormalities with circulatory stasis, hypercoagulable state, endothelial abnormality and hypoperfusion of cerebral blood flow elucidate a strong association between HF and stroke.

Our study demonstrated that the adjusted HR of IS and HS in patients with HF was 1.59 and 1.55 , respectively, which is similar to that reported in studies involving patients from Denmark and Korea. ${ }^{522}$ Kang et al evaluated the risk of IS in 5746 hospitalised patients with $\mathrm{HF}$, which may be contaminated with occult $\mathrm{AF}^{5}{ }^{5}$ Adelborg et al proposed that HF is associated with a short-term risk of IS, but the long-term risk of IS among patients with HF was lower than that in the general population, which may be due to competing mortality. ${ }^{22}$ By matching the propensity scores, our study examined 12179 patients with HF and 12179 patients without HF and found that patients with HF had a significantly higher cumulative incidence of IS than those without HF during the follow-up period (figure 1), which supports the strong association between HF and IS. Moreover, in contrast to containing $10 \%$ of patients with HF with AF or AFL in studies from Korean and Danish cohorts, we excluded AF or AFL in our study and minimised the confounding of AF/AFL and medications with warfarin on the association between HF and IS. ${ }^{522}$ Interestingly, our studies suggested male was a risk factor for IS in the HF group, which was a different outcome compared with Danish and Korean cohorts. Although the Danish cohort indicated that men and women carry equal risk of IS, Korean cohorts revealed that women had a higher risk of IS than men. ${ }^{52}$ Such discrepancies may be attributed to the difference in racial groups participating in these studies.

Our study proposed that diabetes carries a strong adjusted HR of IS in patients with HF, which is consistent with the observation from Melgaard et al. ${ }^{23}$ Several mechanisms explain the occurrence of IS in patients with diabetes. Diabetes impairs NO-mediated vasodilation in vascular endothelium and smooth muscle, resulting in endothelial dysfunction. ${ }^{23-25}$ Additionally, oxidised and glycated low-density lipoproteins in patients with diabetes contribute to the development of atherosclerosis. High levels of hypercoagulable and pro-inflammatory mediators, including plasminogen activator inhibitor-I, interleukin-1, interleukin-6, tumour necrosis factor- $\alpha$, C-reactive proteins, activation of $\mathrm{NF \kappa B}$ and Matrix Metallopeptidase 9, are commonly found in patients with diabetes and atherosclerotic cardiovascular disease. ${ }^{232526}$ Furthermore, the hypercoagulable condition involved in cerebrovascular events is associated with the thrombotic state by increasing platelet activation and the level of all clotting factors. ${ }^{2527}$ The combination of the hypercoagulable condition and 
endothelial dysfunction in patients with $\mathrm{HF}$ and diabetes explains the risk of IS among patients with HF and diabetes.

Our study indicated that the association of IS and HF is significant in patients who have the monthly salary of 15 000-20000 than who have higher income. Blue-collar workers have low income and reside in areas that lack proper sanitation facilities. These people lack awareness about personal health, disease occurrence and disease prevention. The medication compliance is a significant concern among these people. Additionally, medical resources are insufficient in low urbanised areas, which contributes to the elevated risk of stroke in patients with $\mathrm{HF}^{29}{ }^{30}$

\section{Limitations}

Several limitations in our study should be pointed out. First, our NHIRD did not record life habit-related risk factors in detail, including cigarette smoking, alcohol drinking, accurate body mass index, socioeconomic condition and family history. Second, occult AF still could not be totally excluded despite the efforts to exclude all the ICD-9-CM codes of AF and AFL from the index date and follow-up period. Third, the retrospective cohort study is usually lower evidence than the randomised controlled trials because a retrospective cohort study is subject to have many unknown or uncontrolled confounding factors. Last, this study is required a large prospective study to clarify that the benefit of anticoagulants, including warfarin or novel oral anticoagulants, for stroke prevention in patients with HF without AF or AFL. Regardless of above limitations, this study provided significant evidence on the risk of IS among patients with HF without AF or AFL.

\section{CONCLUSION}

In this study, we concluded that HF without AF or AFL is a strong independent risk factor for IS, particularly with old age ( $>65$ years of age), male sex, low income, low urbanisation and diabetes. This study emphasised awareness regarding IS among patients with HF, even without AF or AFL. A large prospective study is warranted to evaluate the use of anticoagulants for such patients.

\section{Key messages}

\section{What is already known about this subject?}

- The risk of ischaemic stroke (IS) among patients with heart failure (HF) in the absence of atrial fibrillation (AF) or atrial flutter (AFL) had not been explored extensively.

\section{What does this study add?}

- This study indicated that HF is a strong independent risk factor for IS, even in the absence of AF or AFL.

\section{How might this impact on clinical practice? \\ - Clinical physicians should investigate IS through routine screening and careful monitoring of patients with HF.}

Contributors All authors have contributed substantially to, and are in agreement with the content of, the manuscript: Conception/Design: Y-LC, C-HK; Provision of study materials: C-HK; Collection and/or assembly of data: Y-LC, C-SL, C-LL, C-HK; Data analysis and interpretation: All authors; Manuscript preparation: All authors; Final approval of manuscript: All authors.

Funding This study is supported by the grants from Tri-Service General Hospita (TSGH-C107-007-007-S02 to C-SL), Ministry of National Defense-Medical Affairs Bureau (MAB-106-082 to C-SL) and Taiwan Ministry of Science and Technology (MOST 106-2314-B-016-038-MY3 to C-SL), Taiwan Ministry of Health and Welfare Clinical Trial Center (MOHW108-TDU-B-212-133004), China Medical University Hospital, Academia Sinica Stroke Biosignature Project (BM10701010021), MOST Clinical Trial Consortium for Stroke (MOST 108-2321-B-039-003-), Tseng-Lien Lin
Foundation, Taichung, Taiwan, and Katsuzo and Kiyo Aoshima Memorial Funds, Japan.

Disclaimer The funders had no role in study design, data collection or analysis, decision to publish or preparation of the manuscript. No additional external funding was received for this study.

Competing interests None declared.

Patient consent for publication Not required.

Ethics approval The Research Ethics Committee of China Medical University and Hospital in Taiwan approved the study (CMUH104-REC2-115-R4).

Provenance and peer review Not commissioned; externally peer reviewed. Data availability statement Data are available upon reasonable request.

ORCID iD

Chia-Hung Kao http://orcid.org/0000-0002-6368-3676

\section{REFERENCES}

1 Kim W, Kim EJ. Heart failure as a risk factor for stroke. J Stroke 2018:20:33-45.

2 Yancy CW, Jessup M, Bozkurt B, et al. 2013 ACCF/AHA guideline for the management of heart failure: a report of the American College of cardiology Foundation/American heart association Task force on practice guidelines. Circulation 2013;128:e240-327.

3 Roger VL. Epidemiology of heart failure. Circ Res 2013;113:646-59.

4 Braunwald E. Shattuck lecture--cardiovascular medicine at the turn of the millennium: triumphs, concerns, and opportunities. N Engl J Med 1997;337:1360-9.

5 Kang S-H, Kim J, Park JJ, et al. Risk of stroke in congestive heart failure with and without atrial fibrillation. Int J Cardiol 2017;248:182-7.

6 Scherbakov N, Haeusler KG, Doehner W. Ischemic stroke and heart failure: facts and numbers. ESC Heart Fail 2015;2:1-4.

7 Haeusler KG, Laufs U, Endres M. Chronic heart failure and ischemic stroke. Stroke 2011;42:2977-82.

8 Sacco RL, Benjamin EJ, Broderick JP, et al. American heart association prevention conference. IV. prevention and rehabilitation of stroke. risk factors. Stroke 1997;28:1507-17.

9 Divani AA, Vazquez G, Asadollahi $M$, et al. Nationwide frequency and association of heart failure on stroke outcomes in the United States. J Card Fail 2009;15:11-16.

10 Pullicino PM, Halperin JL, Thompson JL. Stroke in patients with heart failure and reduced left ventricular ejection fraction. Neurology 2000;54:288-94.

11 Hays AG, Sacco RL, Rundek T, et al. Left ventricular systolic dysfunction and the risk of ischemic stroke in a multiethnic population. Stroke 2006;37:1715-9.

12 Lori S. Parsons ORG, Seattle, Washington. Performing a 1:N Case-Control Match on Propensity Score 2001.

13 Fine JP, Gray RJ. A proportional hazards model for the Subdistribution of a competing risk. J Am Stat Assoc 1999:94:496-509.

14 Wolf PA, Abbott RD, Kannel WB. Atrial fibrillation as an independent risk factor for stroke: the Framingham study. Stroke 1991:22:983-8.

15 Caldwell JC, Mamas MA, Neyses L, et al. What are the thromboembolic risks of heart failure combined with chronic or paroxysmal AF? J Card Fail 2010;16:340-7.

16 Lip GY, Gibbs CR. Does heart failure confer a hypercoagulable state? Virchow's triad revisited. J Am Coll Cardiol 1999:33:1424-6.

17 Jug B, Vene N, Salobir BG, et al. Procoagulant state in heart failure with preserved left ventricular ejection fraction. Int Heart J 2009:50:591-600.

18 Di Tullio MR, Qian M, Thompson JLP, et al. Left ventricular ejection fraction and risk of stroke and cardiac events in heart failure: data from the warfarin versus aspirin in reduced ejection fraction trial. Stroke 2016:47:2031-7.

19 Falk RH, Foster E, Coats MH. Ventricular thrombi and thromboembolism in dilated cardiomyopathy: a prospective follow-up study. Am Heart J 1992:123:136-42.

20 Havakuk 0, King KS, Grazette L, et al. Heart failure-induced brain injury. J Am Coll Cardiol 2017:69:1609-16.

21 Georgiadis D, Sievert M, Cencetti S, et al. Cerebrovascular reactivity is impaired in patients with cardiac failure. Eur Heart J 2000;21:407-13.

22 Adelborg K, Szépligeti S, Sundbøll J, et al. Risk of stroke in patients with heart failure: a population-based 30-year cohort study. Stroke 2017:48:1161-8.

23 Melgaard L, Gorst-Rasmussen A, Søgaard P, et al. Diabetes mellitus and risk of ischemic stroke in patients with heart failure and NO atrial fibrillation. Int J Cardiol 2016:209:1-6

24 Patel SS. Cerebrovascular complications of diabetes: alpha glucosidase inhibitor as potential therapy. Horm Metab Res 2016:48:83-91.

25 Chen R, Ovbiagele B, Feng W. Diabetes and stroke: epidemiology, pathophysiology, pharmaceuticals and outcomes. Am J Med Sci 2016:351:380-6.

26 Vazzana N, Ranalli P, Cuccurullo C, et al. Diabetes mellitus and thrombosis. Thromb Res 2012:129:371-7.

27 Brownlee M. The pathobiology of diabetic complications: a unifying mechanism Diabetes 2005:54:1615-25.

28 Carr ME. Diabetes mellitus: a hypercoagulable state. J Diabetes Complications 2001;15:44-54. 
29 Chu L-M, Liu C-C, Yeh C-C, et al. Increased diabetes risk and interaction with social and medical events in patients upon stroke: two nationwide studies. Atherosclerosis 2017;265:87-92.
30 Leproult R, Holmbäck U, Van Cauter E. Circadian misalignment augments markers of insulin resistance and inflammation, independently of sleep loss. Diabetes 2014;63:1860-9. 